\title{
Evaluation of Substructure Reduction Techniques with Fixed and Free Interfaces
}

\author{
Fabian M. Gruber* - Daniel J. Rixen \\ Technical University of Munich, Institute of Applied Mechanics, Germany
}

\begin{abstract}
Substructure reduction techniques are efficient methods to reduce the size of large models used to analyze the dynamical behavior of complex structures. The most popular approach is a fixed interface method, the Craig-Bampton method (1968), which is based on fixed interface vibration modes and interface constraint modes. In contrast, free interface methods employing free interface vibration modes together with attachment modes are also used, e.g. MacNeal's method (1971) and Rubin's method (1975). The methods mentioned so far assemble the substructures using interface displacements (primal assembly). The dual Craig-Bampton method (2004) uses the same ingredients as the two aforementioned free interface methods, but assembles the substructures using interface forces (dual assembly). This method enforces only weak interface compatibility between the substructures, thereby avoiding interface locking problems as sometimes experienced in the primal assembly approaches using free interface modes. The dual Craig-Bampton method leads to simpler reduced matrices compared to other free interface methods and the reduced matrices are sparse, similar to the classical Craig-Bampton matrices. In this contribution we evaluate the primal (classical) formulation of the Craig-Bampton method, the MacNeal method, the Rubin method and the dual formulation of the CraigBampton method. The presented theory and the comparison between the four substructuring methods will be illustrated on the Benfield truss, on a three-dimensional beam frame and on a two-dimensional solid plane stress problem.
\end{abstract}

Keywords: dynamic substructuring, component mode synthesis, model order reduction, dual assembly, Craig-Bampton method, free interface method

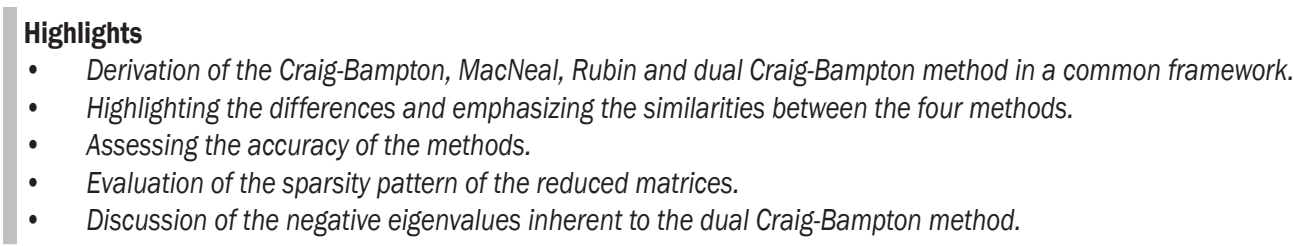

\section{O INTRODUCTION}

Nowadays the increasing performance of modern computers makes it possible to solve very large linear systems of millions of degrees of freedom (DOF). Nevertheless, since dynamic analysis requires solving a lot of linear systems and since the refinement of finite element models is increasing faster than the computing capabilities, dynamic substructuring still remains an essential tool for analyzing dynamical systems in an efficient manner. Building reduced models of subparts of a structure enables sharing models between design groups. Moreover the reduction of the DOF of substructures is also important for building reduced order models for optimization and control. If a single component of a system is changed, only that component needs to be reanalyzed and the system can be analyzed at low additional cost. Thus dynamic substructuring offers a flexible and efficient approach to dynamic analysis.

Dynamic substructuring techniques can be classified in two categories depending on the underlying modes which are used [1]. The term mode can refer to all kind of structural shape vectors. The first class consists of methods using fixed interface vibration modes and interface constraint modes to represent the substructure dynamics. The method commonly used is the Craig-Bampton method (CBM) [2] which assembles the substructures in a primal way using interface displacements in order to enforce interface compatibility. The second class consists of methods using free interface vibration modes and attachment modes. Common representatives of that class are MacNeal's method (MNM) [3] and Rubin's method (RM) [4] using a primal assembly process as well. Herting generalizes in [5] the concept of component mode synthesis to include any kind of interface boundary condition for the modes. In contrast to the aforementioned methods, the dual Craig-Bampton method (DCBM) [6] uses the same ingredients as MacNeal's and Rubin's method, but assembles the substructures in a dual way using interface forces. As a consequence, the DCBM enforces only weak interface compatibility between the substructures, thereby avoiding interface locking problems as sometimes experienced in the primal assembly approaches. Furthermore, the dual CraigBampton method leads to simpler reduced matrices 
compared to other free interface methods and the reduced matrices are sparse, similar to the classical Craig-Bampton matrices. In this contribution we evaluate the primal (classical) formulation of the Craig-Bampton method, the MacNeal method, the Rubin method and the dual formulation of the CraigBampton method. The presented theory and the comparison between the four substructuring methods will be illustrated on three different examples.

In section 1, the differences between primal and dual assembly are stated. Following this, the formulation of the CBM, the MNM, the RM and the DCBM will be outlined in section 2 explaining general properties of the fixed interface method (subsection 2.1) and of the free interface methods (subsection 2.2). These properties will be illustrated subsequently in detail in section 3 using the Benfield Truss (subsection 3.1), a three-dimensional beam frame (subsection 3.2) and a two-dimensional solid plane stress problem (subsection 3.3). Finally a brief summary and conclusions are given in section 4 .

\section{PRIMAL AND DUAL ASSEMBLY OF SUBSTRUCTURES}

Consider a finite element model of a global domain. This domain is divided into $N$ non-overlapping substructures such that every node belongs to exactly one substructure except for the nodes on the interface boundaries. The linear/linearized equation of motion of one substructure is written as

$$
\boldsymbol{M}^{(s)} \ddot{\boldsymbol{u}}^{(s)}+\boldsymbol{K}^{(s)} \boldsymbol{u}^{(s)}=\boldsymbol{f}^{(s)}+\boldsymbol{g}^{(s)},
$$

where the superscript $(s)$ is the label of the particular substructure $(s=1, \ldots, N) . \boldsymbol{M}^{(s)}, \boldsymbol{K}^{(s)}$ and $\boldsymbol{u}^{(s)}$ are the mass matrix, the stiffness matrix and the displacement vector of the substructure, respectively. $\boldsymbol{f}^{(s)}$ is the external force vector and $\boldsymbol{g}^{(s)}$ is the vector of reaction forces on the substructure due to its connection to adjacent substructures at its boundary DOF. The local displacements $\boldsymbol{u}^{(s)}$ of each substructure can be divided in local internal DOF $\boldsymbol{u}_{i}^{(s)}$ and boundary DOF $\boldsymbol{u}_{b}^{(s)}$ :

$$
\boldsymbol{u}^{(s)}=\left[\begin{array}{l}
\boldsymbol{u}_{b}^{(s)} \\
\boldsymbol{u}_{i}^{(s)}
\end{array}\right]=\left[\begin{array}{cc}
\boldsymbol{L}_{b}^{(s)} & \mathbf{0} \\
\mathbf{0} & \boldsymbol{I}
\end{array}\right]\left[\begin{array}{c}
\boldsymbol{u}_{b} \\
\boldsymbol{u}_{i}^{(s)}
\end{array}\right] .
$$

The boundary DOF $\boldsymbol{u}_{b}^{(s)}=\boldsymbol{L}_{b}^{(s)} \boldsymbol{u}_{b}$ of the substructure are a subset of the assembled boundary DOF $\boldsymbol{u}_{b}$ of the global domain. $\boldsymbol{L}_{b}^{(s)}$ is a Boolean localization matrix (connectivity matrix) relating the assembled boundary DOF $\boldsymbol{u}_{b}$ of the global domain to the substructure boundary DOF $\boldsymbol{u}_{b}^{(s)}$. Consequently, the equation of motion, Eq. (1), of one substructure partitioned in the same manner writes

$$
\begin{gathered}
{\left[\begin{array}{cc}
\boldsymbol{M}_{b b}^{(s)} & \boldsymbol{M}_{b i}^{(s)} \\
\boldsymbol{M}_{i b}^{(s)} & \boldsymbol{M}_{i i}^{(s)}
\end{array}\right]\left[\begin{array}{l}
\ddot{\boldsymbol{u}}_{b}^{(s)} \\
\ddot{\boldsymbol{u}}_{i}^{(s)}
\end{array}\right]+\left[\begin{array}{cc}
\boldsymbol{K}_{b b}^{(s)} & \boldsymbol{K}_{b i}^{(s)} \\
\boldsymbol{K}_{i b}^{(s)} & \boldsymbol{K}_{b b}^{(s)}
\end{array}\right]\left[\begin{array}{c}
\boldsymbol{u}_{b}^{(s)} \\
\boldsymbol{u}_{i}^{(s)}
\end{array}\right]=} \\
=\left[\begin{array}{c}
\boldsymbol{f}_{b}^{(s)} \\
\boldsymbol{f}_{i}^{(s)}
\end{array}\right]+\left[\begin{array}{c}
\boldsymbol{g}_{b}^{(s)} \\
\mathbf{0}
\end{array}\right] .
\end{gathered}
$$

Defining the local Boolean localization matrix $\boldsymbol{A}^{(s)}$ which is selecting the boundary DOF of substructure $s$ gives the relation

$$
\boldsymbol{u}_{b}^{(s)}=\boldsymbol{A}^{(s)} \boldsymbol{u}^{(s)},
$$

which will be used later.

\subsection{Primal Assembly}

The Eqs. (1) and (2) of all substructures $N$ can be assembled in a primal way as:

$$
\boldsymbol{M}_{a} \ddot{\boldsymbol{u}}_{a}+\boldsymbol{K}_{a} \boldsymbol{u}_{a}=\boldsymbol{f}_{a},
$$

where

$$
\begin{gathered}
\boldsymbol{M}_{a}=\left[\begin{array}{cccc}
\widehat{\boldsymbol{M}}_{b b} & \widehat{\boldsymbol{M}}_{b i}^{(1)} & \ldots & \widehat{\boldsymbol{M}}_{b i}^{(N)} \\
\widehat{\boldsymbol{M}}_{i b}^{(1)} & \widehat{\boldsymbol{M}}_{i i}^{(1)} & & \mathbf{0} \\
\vdots & & \ddots & \\
\widehat{\boldsymbol{M}}_{i b}^{(N)} & \mathbf{0} & & \widehat{\boldsymbol{M}}_{i i}^{(N)}
\end{array}\right], \\
\boldsymbol{K}_{a}=\left[\begin{array}{cccc}
\widehat{\boldsymbol{K}}_{b b} & \widehat{\boldsymbol{K}}_{b i}^{(1)} & \ldots & \widehat{\boldsymbol{K}}_{b i}^{(N)} \\
\widehat{\boldsymbol{K}}_{i b}^{(1)} & \widehat{\boldsymbol{K}}_{i i}^{(1)} & & \mathbf{0} \\
\vdots & & \ddots & \\
\widehat{\boldsymbol{K}}_{i b}^{(N)} & \mathbf{0} & & \widehat{\boldsymbol{K}}_{i i}^{(N)}
\end{array}\right], \\
\boldsymbol{u}_{a}=\left[\begin{array}{c}
\boldsymbol{u}_{b} \\
\boldsymbol{u}_{i}^{(1)} \\
\vdots \\
\boldsymbol{u}_{i}^{(N)}
\end{array}\right], \\
\boldsymbol{f}_{a}=\left[\begin{array}{c}
\widehat{\boldsymbol{f}}_{b}^{(1)} \\
\vdots \\
\boldsymbol{f}_{i}^{(N)}
\end{array}\right],
\end{gathered}
$$

with

$$
\begin{gathered}
\widehat{\boldsymbol{M}}_{b b}=\sum_{s=1}^{N} \boldsymbol{L}_{b}^{(s)^{T}} \boldsymbol{M}_{b b}^{(s)} \boldsymbol{L}_{b}^{(s)}, \\
\widehat{\boldsymbol{M}}_{i b}=\boldsymbol{M}_{i b}^{(s)} \boldsymbol{L}_{b}^{(s)}, \widehat{\boldsymbol{M}}_{b i}=\boldsymbol{L}_{b}^{(s)^{T}} \boldsymbol{M}_{b i}^{(s)}, \\
\widehat{\boldsymbol{K}}_{b b}=\sum_{s=1}^{N} \boldsymbol{L}_{b}^{(s)^{T}} \boldsymbol{K}_{b b}^{(s)} \boldsymbol{L}_{b}^{(s)}, \\
\widehat{\boldsymbol{K}}_{i b}=\boldsymbol{K}_{i b}^{(s)} \boldsymbol{L}_{b}^{(s)}, \widehat{\boldsymbol{K}}_{b i}=\boldsymbol{L}_{b}^{(s)^{T}} \boldsymbol{K}_{b i}^{(s)}, \\
\widehat{\boldsymbol{f}}_{b}=\sum_{s=1}^{N} \boldsymbol{L}_{b}^{(s)^{T}} \boldsymbol{f}_{b}^{(s)} .
\end{gathered}
$$


The reaction forces $\boldsymbol{g}^{(s)}$ on the interfaces of the substructures cancel out during assembly. This assembly is called primal assembly since the compatibility between the substructures is enforced using the same boundary displacements for adjacent substructures.

\subsection{Dual Assembly}

Another way to enforce the interface compatibility between the substructures is to consider the interface connecting forces as unknowns. These forces must be determined to satisfy the interface compatibility condition (displacement equality) and the local equation of motion of the substructures:

$$
\begin{gathered}
\sum_{s=1}^{N} \boldsymbol{B}^{(s)} \boldsymbol{u}^{(s)}=\mathbf{0} \\
\boldsymbol{M}^{(s)} \ddot{\boldsymbol{u}}^{(s)}+\boldsymbol{K}^{(s)} \boldsymbol{u}^{(s)}+\boldsymbol{B}^{(s)^{T}} \boldsymbol{\lambda}=\boldsymbol{f}^{(s)} .
\end{gathered}
$$

$\boldsymbol{B}^{(s)}$ is a signed Boolean matrix (constraint matrix) acting on the substructure interface DOF. $\boldsymbol{B}^{(s)^{T}} \lambda$ is representing the interconnecting forces between substructures which is corresponding to the negative interface reaction force vector $\boldsymbol{g}^{(s)}$ in Eq. (1) meaning

$$
\boldsymbol{g}^{(s)}=-\boldsymbol{B}^{(s)^{T}} \lambda, \quad \boldsymbol{g}_{b}^{(s)}=-\boldsymbol{B}_{b}^{(s)^{T}} \lambda,
$$

and $\lambda$ is the vector of all Lagrange multipliers acting on the interfaces which are the additional unknowns. The displacement vector $\boldsymbol{u}^{(s)}$ is partitioned according to Eq. (2). With the block-diagonal matrices

$$
\begin{gathered}
\boldsymbol{M}=\left[\begin{array}{ccc}
\boldsymbol{M}^{(1)} & & \mathbf{0} \\
& \ddots & \\
\mathbf{0} & & \boldsymbol{M}^{(N)}
\end{array}\right], \\
\boldsymbol{K}=\left[\begin{array}{ccc}
\boldsymbol{K}^{(1)} & & \mathbf{0} \\
& \ddots & \\
\mathbf{0} & & \boldsymbol{K}^{(N)}
\end{array}\right],
\end{gathered}
$$

and

$$
\begin{gathered}
\boldsymbol{u}=\left[\begin{array}{c}
\boldsymbol{u}^{(1)} \\
\vdots \\
\boldsymbol{u}^{(N)}
\end{array}\right], \quad \boldsymbol{f}=\left[\begin{array}{c}
\boldsymbol{f}^{(1)} \\
\vdots \\
\boldsymbol{f}^{(N)}
\end{array}\right], \\
\boldsymbol{B}=\left[\begin{array}{lll}
\boldsymbol{B}^{(1)} & \cdots & \boldsymbol{B}^{(N)}
\end{array}\right],
\end{gathered}
$$

the set of Eqs. (9) and (10) can be written as:

$$
\left[\begin{array}{cc}
\boldsymbol{M} & \mathbf{0} \\
\mathbf{0} & \mathbf{0}
\end{array}\right]\left[\begin{array}{c}
\ddot{\boldsymbol{u}} \\
\ddot{\lambda}
\end{array}\right]+\left[\begin{array}{cc}
\boldsymbol{K} & \boldsymbol{B}^{T} \\
\boldsymbol{B} & \mathbf{0}
\end{array}\right]\left[\begin{array}{l}
\boldsymbol{u} \\
\lambda
\end{array}\right]=\left[\begin{array}{l}
\boldsymbol{f} \\
\mathbf{0}
\end{array}\right]
$$

In this hybrid formulation the Lagrange multipliers $\lambda$ enforce the interface compatibility constraints and can be identified as interface forces [6]. The dual assembled system in Eq. (16) is equivalent to the primal assembled system in Eq. (5) since both systems express the same local equilibrium for each substructure and enforce the same interface compatibility.

\section{COMPONENT REDUCTION METHODS}

\subsection{Craig-Bampton Method (CBM)}

Considering the partitioned equation of motion in Eq. (3), the internal DOF $\boldsymbol{u}_{i}^{(s)}$ of every substructure can be seen as being excited by its boundary DOF $\boldsymbol{u}_{b}^{(s)}$, namely

$$
\boldsymbol{M}_{i i}^{(s)} \ddot{\boldsymbol{u}}_{i}^{(s)}+\boldsymbol{K}_{i i}^{(s)} \boldsymbol{u}_{i}^{(s)}=\boldsymbol{f}_{i}^{(s)}-\boldsymbol{K}_{i b}^{(s)} \boldsymbol{u}_{b}^{(s)}-\boldsymbol{M}_{i b}^{(s)} \ddot{\boldsymbol{u}}_{b}^{(s)} .
$$

This indicates that $\boldsymbol{u}_{i}^{(s)}$ of each substructure can be approximated by a superposition of a static response and of eigenmodes associated to $\boldsymbol{M}_{i i}^{(s)}$ and $\boldsymbol{K}_{i i}^{(s)}$. The static response is given by

$$
\boldsymbol{u}_{i, s t a t}^{(s)}=-\boldsymbol{K}_{i i}^{(s)^{-1}} \boldsymbol{K}_{i b}^{(s)} \boldsymbol{u}_{b}^{(s)}=\Psi_{i b}^{(s)} \boldsymbol{u}_{b}^{(s)},
$$

where the columns of matrix $\Psi_{i b}^{(s)}$ are the static response modes also called constraint modes [2]. The fixed interface normal modes $\phi_{k}^{(s)}$ are obtained as eigensolutions of the generalized eigenproblem $\boldsymbol{K}_{i i}^{(s)} \boldsymbol{\phi}_{k}^{(s)}=\omega_{k}^{(s)^{2}} \boldsymbol{M}_{i i}^{(s)} \boldsymbol{\phi}_{k}^{(s)}$. The columns of the $n_{i}^{(s)} \times n_{\phi}^{(s)}$ matrix $\Phi_{i \phi}^{(s)}$ contain the first $n_{\phi}^{(s)}$ fixed interface normal modes $\phi_{k}^{(s)}$ which can also be considered as the free vibration modes of the substructure $s$ clamped on its boundary DOF $\boldsymbol{u}_{b}^{(s)}$. The approximation of $\boldsymbol{u}_{i}^{(s)}$ therefore writes

$$
\boldsymbol{u}_{i}^{(s)} \approx \boldsymbol{u}_{i, s t a t}^{(s)}+\boldsymbol{\Phi}_{i \phi}^{(s)} \boldsymbol{\eta}^{(s)},
$$

and the displacements of the substructure are approximated by

$$
\left[\begin{array}{c}
\boldsymbol{u}_{b}^{(s)} \\
\boldsymbol{u}_{i}^{(s)}
\end{array}\right]=\left[\begin{array}{cc}
\boldsymbol{I}_{b b}^{(s)} & \mathbf{0}_{b \phi}^{(s)} \\
\boldsymbol{\Psi}_{i b}^{(s)} & \boldsymbol{\Phi}_{i \phi}^{(s)}
\end{array}\right]\left[\begin{array}{l}
\boldsymbol{u}_{b}^{(s)} \\
\boldsymbol{\eta}^{(s)}
\end{array}\right],
$$

with the vector of modal parameters $\eta^{(s)}$ of dimension $n_{\phi}^{(s)}$ corresponding to the amplitudes of the fixed interface normal modes $\Phi_{i \phi}^{(s)}$. The CBM reduction matrix $\boldsymbol{T}_{C B}$ for reducing the primal assembled system can be defined as: 


$$
\boldsymbol{u}_{a} \approx \underbrace{\left[\begin{array}{cccc}
\boldsymbol{I} & \mathbf{0} & \cdots & \mathbf{0} \\
\boldsymbol{\Psi}_{i b}^{(1)} \boldsymbol{L}_{b}^{(1)} & \boldsymbol{\Phi}_{i \phi}^{(1)} & & \mathbf{0} \\
\vdots & & \ddots & \\
\boldsymbol{\Psi}_{i b}^{(N)} \boldsymbol{L}_{b}^{(N)} & \mathbf{0} & & \boldsymbol{\Phi}_{i \phi}^{(N)}
\end{array}\right]}_{\boldsymbol{T}_{C B}}\left[\begin{array}{c}
\boldsymbol{u}_{b} \\
\boldsymbol{\eta}^{(1)} \\
\vdots \\
\boldsymbol{\eta}^{(N)}
\end{array}\right],
$$

and the CBM reduced matrices

$$
\begin{aligned}
\boldsymbol{K}_{\text {red }, C B} & =\boldsymbol{T}_{C B}^{T} \boldsymbol{K}_{a} \boldsymbol{T}_{C B}, \\
\boldsymbol{M}_{\text {red }, C B} & =\boldsymbol{T}_{C B}^{T} \boldsymbol{M}_{a} \boldsymbol{T}_{C B},
\end{aligned}
$$

are found in [2].

\subsection{Free Interface Methods}

Considering the equation of motion (Eq. (10)) of substructure $s$, every substructure can be seen as being excited by the interface connection forces and the external forces. This indicates that the displacements of each substructure $\boldsymbol{u}^{(s)}$ can be expressed in terms of local static solutions $\boldsymbol{u}_{\text {stat }}^{(s)}$ and in terms of eigenmodes associated to the entire substructure matrices $\boldsymbol{K}^{(s)}$ and $\boldsymbol{M}^{(s)}$ :

$$
\boldsymbol{u}^{(s)}=\boldsymbol{u}_{\text {stat }}^{(s)}+\sum_{j=1}^{n^{(s)}-r^{(s)}} \boldsymbol{\theta}_{j}^{(s)} \eta_{j}^{(s)}
$$

with the static solution

$$
\boldsymbol{u}_{\text {stat }}^{(s)}=-\boldsymbol{K}^{(s)^{+}} \boldsymbol{B}^{(s)^{T}} \lambda+\sum_{j=1}^{r^{(s)}} \boldsymbol{R}_{j}^{(s)} \alpha_{j}^{(s)} .
$$

The static response $\boldsymbol{u}_{\text {stat }}^{(s)}$ is obtained by solving Eq. (10) under the assumption of no inertia forces and no external forces acting on the substructure. $\boldsymbol{K}^{\left(s^{+}\right.}$is equal to the inverse of $\boldsymbol{K}^{(s)}$ when there are enough boundary conditions to prevent the substructure from floating when its interface with neighboring substructures is free [6]. If a substructure is floating, $\boldsymbol{K}^{(s)^{+}}$is a generalized inverse of $\boldsymbol{K}^{(s)}$ and $\boldsymbol{R}^{(s)}$ is the matrix containing the $r^{(s)}$ rigid body modes as columns. The vector $\alpha^{(s)}$ contains the amplitudes $\alpha_{j}^{(s)}$ of the rigid body modes and the vector $\eta^{(s)}$ contains the amplitudes $\eta_{j}^{(s)}$ of the local eigenmodes $\boldsymbol{\theta}_{j}^{(s)}$ being eigensolutions of the generalized eigenproblem $\boldsymbol{K}^{(s)} \boldsymbol{\theta}_{j}^{(s)}=\omega_{j}^{(s)^{2}} \boldsymbol{M}^{(s)} \boldsymbol{\theta}_{j}^{(s)}$. An approximation is obtained by retaining only the first $n_{\theta}^{(s)}$ free interface normal modes $\boldsymbol{\theta}_{j}^{(s)}$. Calling $\Theta^{(s)}$ the matrix containing only these $n_{\theta}^{(s)}$ eigenmodes, the approximation of the displacements $\boldsymbol{u}^{(s)}$ of the substructure is given by:

$$
\boldsymbol{u}^{(s)} \approx-\boldsymbol{K}^{(s)^{+}} \boldsymbol{B}^{(s)^{T}} \boldsymbol{\lambda}+\boldsymbol{R}^{(s)} \boldsymbol{\alpha}^{(s)}+\Theta^{(s)} \boldsymbol{\eta}^{(s)} .
$$

Since a part of the subspace spanned by $\Theta^{(s)}$ is already included in $\boldsymbol{K}^{(s)^{+}}$the residual flexibility matrix $\boldsymbol{G}_{r}^{(s)}$ can be used instead of $\boldsymbol{K}^{(s)^{+}}$, which is defined by:

$$
\boldsymbol{G}_{r}^{(s)}=\sum_{j=n_{\theta}^{(s)}+1}^{n^{(s)}-r_{j}^{(s)}} \frac{\boldsymbol{\theta}_{j}^{(s)} \boldsymbol{\theta}_{j}^{(s)^{T}}}{\omega_{j}^{(s)^{2}}}=\boldsymbol{K}^{(s)^{+}}-\sum_{j=1}^{n_{\theta}^{(s)}} \frac{\boldsymbol{\theta}_{j}^{(s)} \boldsymbol{\theta}_{j}^{(s)^{T}}}{\omega_{j}^{(s)}} .
$$

Note that, by construction $\boldsymbol{G}_{r}^{(s)}=\boldsymbol{G}_{r}^{(s)^{T}}$, which is computed using the second equality in Eq. (27). For further properties of $\boldsymbol{G}_{r}^{(s)}$ see [6]. As a result the approximation of one substructure writes:

$$
\begin{aligned}
\boldsymbol{u}^{(s)} & \approx\left[\begin{array}{lll}
\boldsymbol{R}^{(s)} & \Theta^{(s)} & -\boldsymbol{G}_{r}^{(s)} \boldsymbol{B}^{(s)^{T}}
\end{array}\right]\left[\begin{array}{c}
\boldsymbol{\alpha}^{(s)} \\
\eta^{(s)} \\
\lambda
\end{array}\right]= \\
& =\underbrace{\left[\begin{array}{lll}
\boldsymbol{R}^{(s)} & \Theta^{(s)} & \boldsymbol{G}_{r}^{(s)} \boldsymbol{A}^{(s)^{T}}
\end{array}\right]}_{\boldsymbol{T}_{1}^{(s)}}\left[\begin{array}{c}
\boldsymbol{\alpha}^{(s)} \\
\boldsymbol{\eta}^{(s)} \\
\boldsymbol{g}_{b}^{(s)}
\end{array}\right] .
\end{aligned}
$$

$\boldsymbol{G}_{r}^{(s)} \boldsymbol{A}^{(s)^{T}}$ is the matrix containing the residual flexibility attachment modes of substructure $s$, since the Boolean localization matrix $\boldsymbol{A}^{(s)^{T}}$ as defined in Eq. (4) simply picks the columns of $\boldsymbol{G}_{r}^{(s)}$ associated to the boundary DOF [7]. The approximation in Eq. (28) can now be used to reduce the substructure DOF. Using the orthogonality properties of the modes in Eq. (28) the equation of motion of one substructure in Eq. (1) becomes

$$
\boldsymbol{M}_{\text {free }}^{(s)}\left[\begin{array}{c}
\ddot{\alpha}^{(s)} \\
\ddot{\boldsymbol{\eta}}^{(s)} \\
\ddot{\boldsymbol{g}}_{b}^{(s)}
\end{array}\right]+\boldsymbol{K}_{\text {friee }}^{(s)}\left[\begin{array}{c}
\boldsymbol{\alpha}^{(s)} \\
\boldsymbol{\eta}^{(s)} \\
\boldsymbol{g}_{b}^{(s)}
\end{array}\right]=\boldsymbol{T}_{1}^{(s)^{T}}\left(\boldsymbol{f}^{(s)}+\boldsymbol{g}^{(s)}\right),
$$

with the matrices

$$
\begin{gathered}
\boldsymbol{M}_{\text {free }}^{(s)}=\boldsymbol{T}_{1}^{(s)^{T}} \boldsymbol{M}^{(s)} \boldsymbol{T}_{1}^{(s)}=\left[\begin{array}{ccc}
\boldsymbol{I} & \mathbf{0} & \mathbf{0} \\
\mathbf{0} & \boldsymbol{I} & \mathbf{0} \\
\mathbf{0} & \mathbf{0} & \boldsymbol{M}_{r, b b}^{(s)}
\end{array}\right], \\
\boldsymbol{K}_{\text {free }}^{(s)}=\boldsymbol{T}_{1}^{(s)^{T}} \boldsymbol{K}^{(s)} \boldsymbol{T}_{1}^{(s)}=\left[\begin{array}{ccc}
\mathbf{0} & \mathbf{0} & \mathbf{0} \\
\mathbf{0} & \boldsymbol{\Omega}^{(s)^{2}} & \mathbf{0} \\
\mathbf{0} & \mathbf{0} & \boldsymbol{G}_{r, b b}^{(s)}
\end{array}\right], \\
\boldsymbol{G}_{r, b b}^{(s)}=\boldsymbol{A}^{(s)} \boldsymbol{G}_{r}^{(s)} \boldsymbol{A}^{(s)^{T}}, \\
\boldsymbol{M}_{r, b b}^{(s)}=\boldsymbol{A}^{(s)} \boldsymbol{G}_{r}^{(s)} \boldsymbol{M}^{(s)} \boldsymbol{G}_{r}^{(s)} \boldsymbol{A}^{(s)^{T}} .
\end{gathered}
$$


$\boldsymbol{G}_{r, b b}^{(s)}$ is the residual flexibility and $\boldsymbol{M}_{r, b b}^{(s)}$ is the interface inertia associated to the residual flexibility related to the boundary DOF, respectively, and $\boldsymbol{\Omega}^{(s)}$ being a diagonal matrix containing the remaining $n_{\theta}^{(s)}$ eigenvalues $\omega_{j}^{(s)}$.

\subsubsection{Rubin Method (RM)}

In order to assemble the substructure equation of motion in Eq. (29) in the global system a second transformation is applied by the RM [4]. The force DOF $\boldsymbol{g}_{b}^{(s)}$ are transformed back to the boundary displacements $\boldsymbol{u}_{b}^{(s)}$ using Eq. (28) [7]:

$$
\boldsymbol{u}_{b}^{(s)}=\boldsymbol{A}^{(s)} \boldsymbol{u}^{(s)}=\boldsymbol{R}_{b}^{(s)} \boldsymbol{\alpha}^{(s)}+\Theta_{b}^{(s)} \eta^{(s)}+\boldsymbol{G}_{r, b b}^{(s)} \boldsymbol{g}_{b}^{(s)} .
$$

$\boldsymbol{R}_{b}^{(s)}$ and $\Theta_{b}^{(s)}$ are the subparts of $\boldsymbol{R}^{(s)}$ and $\Theta^{(s)}$ related to the boundary DOF, respectively. From this equation the interface force DOF can be solved as:

$$
\boldsymbol{g}_{b}^{(s)}=\boldsymbol{K}_{r, b b}^{(s)}\left(\boldsymbol{u}_{b}^{(s)}-\boldsymbol{R}_{b}^{(s)} \boldsymbol{\alpha}^{(s)}-\Theta_{b}^{(s)} \boldsymbol{\eta}^{(s)}\right),
$$

with $\boldsymbol{K}_{r, b b}^{(s)}=\boldsymbol{G}_{r, b b}^{(s)^{-1}}$. The transformation matrix $\boldsymbol{T}_{2}^{(s)}$ from force DOF $\boldsymbol{g}_{b}^{(s)}$ back to the boundary displacements $\boldsymbol{u}_{b}^{(s)}$ leaving $\boldsymbol{\alpha}^{(s)}$ and $\boldsymbol{\eta}^{(s)}$ unchanged writes then:

$$
\boldsymbol{T}_{2}^{(s)}=\left[\begin{array}{ccc}
\boldsymbol{I} & \mathbf{0} & \mathbf{0} \\
\mathbf{0} & \boldsymbol{I} & \mathbf{0} \\
-\boldsymbol{K}_{r, b b}^{(s)} \boldsymbol{R}_{b}^{(s)} & -\boldsymbol{K}_{r, b b}^{(s)} \Theta_{b}^{(s)} & \boldsymbol{K}_{r, b b}^{(s)}
\end{array}\right] .
$$

Application of this transformation to the matrices of Eqs. (30) and (31) gives the RM reduced matrices of one substructure

$$
\begin{gathered}
\boldsymbol{K}_{\text {red }, R}^{(s)}=\boldsymbol{T}_{2}^{(s)^{T}} \boldsymbol{K}_{\text {free }}^{(s)} \boldsymbol{T}_{2}^{(s)}, \\
\boldsymbol{M}_{\text {red }, R}^{(s)}=\boldsymbol{T}_{2}^{(s)^{T}} \boldsymbol{M}_{\text {free }}^{(s)} \boldsymbol{T}_{2}^{(s)} .
\end{gathered}
$$

The RM reduction matrix for one substructure writes therefore:

$$
\boldsymbol{T}_{R}^{(s)}=\boldsymbol{T}_{1}^{(s)} \boldsymbol{T}_{2}^{(s)},
$$

and the RM reduced matrices

$$
\begin{gathered}
\boldsymbol{K}_{\text {red }, R}^{(s)}=\boldsymbol{T}_{R}^{(s)^{T}} \boldsymbol{K}^{(s)} \boldsymbol{T}_{R}^{(s)}, \\
\boldsymbol{M}_{\text {red }, R}^{(s)}=\boldsymbol{T}_{R}^{(s)^{T}} \boldsymbol{M}^{(s)} \boldsymbol{T}_{R}^{(s)},
\end{gathered}
$$

are found [7]. These matrices can be directly assembled using primal assembly to get the RM reduced matrices $\boldsymbol{K}_{\text {red,R }}$ and $\boldsymbol{M}_{\text {red }, R}$ of the global system. This process was outlined in section 1.1 and applied in section 2.1 for the CBM. The RM applies the reduction matrix $\boldsymbol{T}_{R}^{(s)}$ consistently to the mass and stiffness matrix resulting in a true Rayleigh-Ritz method as was observed in [8].

\subsubsection{MacNeal Method (MNM)}

The MNM [3] is nearly identical to the RM except for a small change. First we will derive the preliminary MNM reduced matrices $\tilde{\boldsymbol{K}}_{\text {red, }, M N}^{(s)}$ and $\tilde{\boldsymbol{M}}_{\text {red }, M N}^{(s)}$ following the derivation of the RM to show the similarities between these two methods. The reduced stiffness matrix of both the RM and the MNM are identical (given in Eq. (40))

$$
\tilde{\boldsymbol{K}}_{r e d, M N}^{(s)}=\boldsymbol{K}_{r e d, R}^{(s)},
$$

but the MNM reduced mass matrix $\tilde{\boldsymbol{M}}_{\text {red }, M N}^{(s)}$ is obtained differently. The residual mass term $\boldsymbol{M}_{r, b b}^{(s)}$ of the matrix $\boldsymbol{M}_{\text {free }}^{(s)}$ in Eq. (30) is neglected resulting in a modified matrix labeled

$$
\boldsymbol{M}_{\text {free }, M N}^{(s)}=\left[\begin{array}{lll}
\boldsymbol{I} & \mathbf{0} & \mathbf{0} \\
\mathbf{0} & \boldsymbol{I} & \mathbf{0} \\
\mathbf{0} & \mathbf{0} & \mathbf{0}
\end{array}\right],
$$

instead of $\boldsymbol{M}_{\text {free }}^{(s)}$ for the MNM [7]. The preliminary MNM reduced mass matrix writes now:

$$
\tilde{\boldsymbol{M}}_{\text {red }, M N}^{(s)}=\boldsymbol{T}_{2}^{(s)^{T}} \boldsymbol{M}_{\text {free, } M N}^{(s)} \boldsymbol{T}_{2}^{(s)}=\boldsymbol{M}_{\text {free }, M N}^{(s)} .
$$

This gives in fact inconsistent equations of motion since the mass and stiffness matrices are not reduced with the same basis. The assembly of the MNM reduced matrices $\tilde{\boldsymbol{K}}_{\text {red,MN }}^{(s)}$ and $\tilde{\boldsymbol{M}}_{\text {red,MN }}^{(s)}$ in the global system proceeds in the same manner as for the RM. Observing that the boundary DOF $\boldsymbol{u}_{b}$ have no associated inertia in Eq. (44), those DOF can be condensed out of the equation of motion of the assembled problem and the final MNM reduced matrices $\boldsymbol{K}_{r e d, M N}$ and $\boldsymbol{M}_{\text {red, }, M N}$ are obtained [3]. Thus the size of the assembled MNM system is reduced further by the number of DOF of $\boldsymbol{u}_{b}$.

\subsubsection{Dual Craig-Bampton Method (DCBM)}

Replacing $\boldsymbol{g}_{b}^{(s)}$ according to Eq. (11) by the Lagrange multipliers $\lambda$ in the equation of motion of one substructure in Eq. (29), the reduced substructure matrices can be directly coupled using the dual assembly procedure [6] as outlined in section 1.2. Assembling all substructures $N$ in a dual fashion by keeping the interface forces $\lambda$ as unknowns, the entire structure can consequently be approximated by 


$$
\left[\begin{array}{c}
\boldsymbol{u} \\
\lambda
\end{array}\right] \approx \boldsymbol{T}_{D C B}\left[\begin{array}{c}
\alpha^{(1)} \\
\eta^{(1)} \\
\vdots \\
\alpha^{(N)} \\
\eta^{(N)} \\
\lambda
\end{array}\right]
$$

with the DCBM reduction matrix $\boldsymbol{T}_{D C B}$ :

$\boldsymbol{T}_{D C B}=\left[\begin{array}{cccccc}\boldsymbol{R}^{(1)} & \Theta^{(1)} & & \mathbf{0} & \mathbf{0} & -\boldsymbol{G}_{r}^{(1)} \boldsymbol{B}^{(1) T} \\ \vdots & \vdots & \ddots & \vdots & \vdots & \vdots \\ \mathbf{0} & \mathbf{0} & & \boldsymbol{R}^{(N)} & \Theta^{(N)} & -\boldsymbol{G}_{r}^{(N)} \boldsymbol{B}^{(N) T} \\ \mathbf{0} & \mathbf{0} & & \mathbf{0} & \mathbf{0} & \boldsymbol{I}\end{array}\right] .(46)$

The approximation of the dynamic equations of the dual assembled system in Eq. (16) is

$$
\boldsymbol{M}_{\text {red }, D C B}\left[\begin{array}{c}
\boldsymbol{\alpha}^{(1)} \\
\boldsymbol{\eta}^{(1)} \\
\vdots \\
\boldsymbol{\alpha}^{(N)} \\
\boldsymbol{\eta}^{(N)} \\
\lambda
\end{array}\right]+\boldsymbol{K}_{r e d, D C B}\left[\begin{array}{c}
\boldsymbol{\alpha}^{(1)} \\
\boldsymbol{\eta}^{(1)} \\
\vdots \\
\boldsymbol{\alpha}^{(N)} \\
\boldsymbol{\eta}^{(N)} \\
\lambda
\end{array}\right]=\boldsymbol{T}_{D C B}^{T} \boldsymbol{f},
$$

with the DCBM reduced mass and stiffness matrix

$$
\begin{gathered}
\boldsymbol{M}_{r e d, D C B}=\boldsymbol{T}_{D C B}^{T}\left[\begin{array}{cc}
\boldsymbol{M} & \mathbf{0} \\
\mathbf{0} & \mathbf{0}
\end{array}\right] \boldsymbol{T}_{D C B}=\left[\begin{array}{cc}
\boldsymbol{I} & \mathbf{0} \\
\mathbf{0} & \boldsymbol{M}_{r}
\end{array}\right], \\
\boldsymbol{K}_{r e d, D C B}=\boldsymbol{T}_{D C B}^{T}\left[\begin{array}{cc}
\boldsymbol{K} & \boldsymbol{B}^{T} \\
\boldsymbol{B} & \mathbf{0}
\end{array}\right] \boldsymbol{T}_{D C B},
\end{gathered}
$$

with

$$
\boldsymbol{M}_{r}=\sum_{s=1}^{N} \boldsymbol{B}^{(s)} \boldsymbol{G}_{r}^{(s)} \boldsymbol{M}^{(s)} \boldsymbol{G}_{r}^{(s)} \boldsymbol{B}^{(s)^{T}} .
$$

$\boldsymbol{M}_{\text {red, }, D C B}$ and $\boldsymbol{K}_{\text {red, }, D C B}$ are diagonal for the parts related to the different substructures. The coupling between the substructures is only achieved by the rows and columns related to $\lambda$. The DCBM applies the reduction matrix $\boldsymbol{T}_{D C B}$ consistently to the mass and stiffness matrix resulting in a true Rayleigh-Ritz method.

The DCBM enforces only a weak compatibility between the substructures and does not enforce a strong displacement compatibility between the interfaces compared to many other common reduction methods [6]. Considering the system of Eqs. (9) and (10) multiplied by the reduction matrix $\boldsymbol{T}_{D C B}^{T}$, the last row of Eq. (47) results from

$$
\left[\begin{array}{c}
\boldsymbol{M}^{(1)} \ddot{\boldsymbol{u}}^{(1)}+\boldsymbol{K}^{(1)} \boldsymbol{u}^{(1)}+\boldsymbol{B}^{(1)^{T}} \boldsymbol{\lambda}=\boldsymbol{f}^{(1)} \\
\vdots \\
\boldsymbol{M}^{(N)} \ddot{\boldsymbol{u}}^{(N)}+\boldsymbol{K}^{(N)} \boldsymbol{u}^{(N)}+\boldsymbol{B}^{(N)^{T}} \lambda=\boldsymbol{f}^{(N)} \\
\sum_{s=1}^{N} \boldsymbol{B}^{(s)} \boldsymbol{u}^{(s)}=\mathbf{0}
\end{array}\right],
$$

multiplied from left by the last row of $\boldsymbol{T}_{D C B}^{T}$ which is

$$
\left[\begin{array}{llll}
-\boldsymbol{B}^{(1)} \boldsymbol{G}_{r}^{(1)} & \cdots & -\boldsymbol{B}^{(N)} \boldsymbol{G}_{r}^{(N)} & \boldsymbol{I}
\end{array}\right] .
$$

Replacing the strong interface compatibility condition of Eq. (9) by the weak form according to the multiplication of Eq. (51) by Eq. (52) can be interpreted as follows. Denote $\Delta \boldsymbol{f}^{(s)}$ the residual forces of substructure $s$ resulting from the weak satisfaction of the local equilibrium of the substructure approximating the dynamics by a small number of free interface normal modes. Name $\Delta \boldsymbol{u}^{(s)}=\boldsymbol{G}_{r}^{(s)} \Delta \boldsymbol{f}^{(s)}$ the displacements these residual force $\Delta \boldsymbol{f}^{(s)}$ would create locally. Then the weak interface compatibility condition (Eqs. (51) and (52)) states that a compatibility error (i.e. an interface displacement jump) equal to the incompatibility of $\Delta \boldsymbol{u}^{(s)}$ is permitted [6]. Compared to MacNeal's and Rubin's method [3] and [4], the weak interface compatibility of the DCBM avoids locking problems occurring during the application of the aforementioned methods. Therefore, the approximation accuracy is improved [6]. But the fact that a weak interface compatibility is allowed in the DCBM implies that the infinite eigenvalues related to the Lagrange multipliers $\lambda$ in the non-reduced problem in Eq. (16) are now becoming finite and negative [9]. In practice those negative eigensolutions will appear only in the higher eigenvalue spectrum if the reduction space is rich enough [9]. Nevertheless, the reduction basis has to be selected with care avoiding potential non-physical effects of the possibly occurring negative eigenvalues.

If $\boldsymbol{M}_{r}$ in Eq. (48) is neglected strong interface compatibility is enforced again and the DCBM reduced system with $\boldsymbol{M}_{r}=\mathbf{0}$ is equivalent to the MNM [6]. Then static condensation can be applied again to remove $\lambda$ (as it was done for $\boldsymbol{u}_{b}$ at the end of the derivation of the MNM in section 2.2.2) from the assembled system since no mass is associated. Thus the size of the assembled system is reduced again by the number of DOF of $\lambda$. 


\section{EXAMPLES AND DISCUSSION}

\subsection{Benfield Truss}

The Benfield truss [10] of Fig. 1 is used to compare the results obtainable by the CBM, the MNM, the RM and the DCBM. The planar truss consists of two substructures having uniform bay section whereas all members have constant area and uniform stiffness and mass properties. The left component consists of five equal bays and has a total of 18 joints and the right component consists of four equal bays and has a total of 15 joints [10]. The lowest eigenfrequencies $\omega$ of the entire structure shall be approximated by the different methods. The relative error $\varepsilon_{\text {rel }, j}=\left|\omega_{\text {red }, j}-\omega_{\text {full }, j}\right| / \omega_{\text {full }, j}$ of the $j^{\text {th }}$ eigenfrequency is used as a criterion to assess the accuracy of the different methods. Thereby $\omega_{\text {full, } j}$ is the $j^{\text {th }}$ eigenfrequency of the full (non-reduced) system and $\omega_{\text {red,j }}$ represents the $j^{\text {th }}$ eigenfrequency of the reduced system obtained by each method. Using 5 elastic (fixed or free interface normal modes) per substructure the relative errors $\varepsilon_{r e l}$ depicted in the semi-log graph in Fig. 2 are resulting.
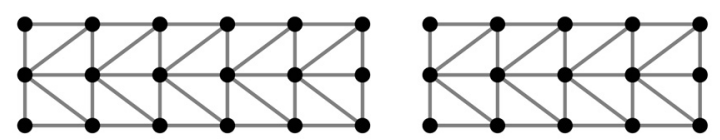

Fig. 1. Benfield truss [10]

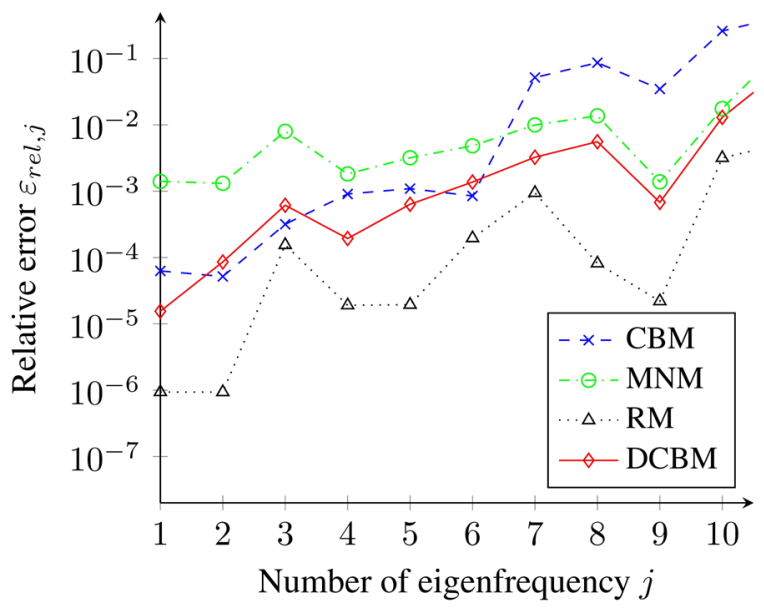

Fig. 2. Relative error of eigenfrequency using 5 normal modes per substructure for the approximation of the lowest eigenfrequencies of the Benfield truss

Since all methods give the correct rigid body modes only the relative errors of the elastic modes are plotted. All methods give a relative error less than $1 \%$ for the first six eigenfrequencies. Comparing the free interface methods for this example, the RM performs always better than the DCBM and the DCBM performs again always better as the MNM. The CBM and the DCBM result in similar frequency errors.

The sparsity pattern of the reduced stiffness matrix $\boldsymbol{K}_{\text {red }}$ and reduced mass matrix $\boldsymbol{M}_{\text {red,MN }}$ of the CBM (Fig. 3), the MNM (Fig. 5), the RM (Fig. 6) and the DCBM (Fig. 4), respectively, illustrate the differences of the assembled reduced structures. Both the reduced stiffness matrix $\boldsymbol{K}_{\text {red }}$ and the reduced mass matrix $\boldsymbol{M}_{\text {red }}$ applying the CBM and the DCBM, respectively, have only diagonal entries for the subparts of each substructure. On the one hand the coupling between the substructures using the CBM is entirely achieved by the last rows and last columns in the mass matrix $\boldsymbol{M}_{r e d, C B}$ (Fig. 3b) and the remaining part is diagonal [2]. On the other hand the coupling applying the DCBM is entirely achieved by the last rows and last columns in the stiffness matrix $\boldsymbol{K}_{\text {red,DCB }}$ (Fig. 4a) and again the remaining part is diagonal [6]. The corresponding degrees of freedoms are either the interface displacements $\boldsymbol{u}_{b}$ or the interface forces $\lambda$ but no direct coupling between the modal parameters of adjacent substructures occurs which ensures the sparse structure.

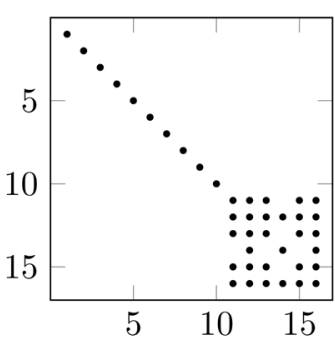

a) $\boldsymbol{K}_{\text {red, } C B}$

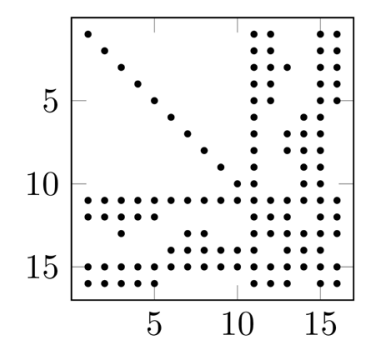

b) $\boldsymbol{M}_{r e d, C B}$
Fig. 3. Sparsity pattern of the reduced matrices applying the CBM using 5 normal modes per substructure

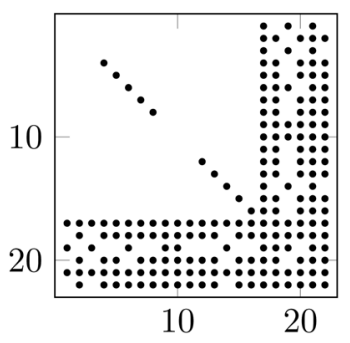

a) $\boldsymbol{K}_{r e d, D C B}$

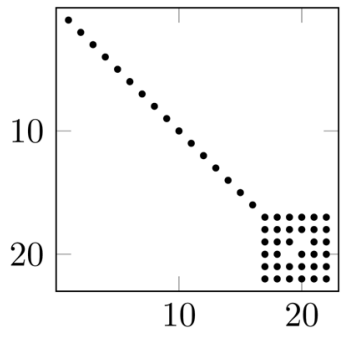

b) $\boldsymbol{M}_{r e d, D C B}$
Fig. 4. Sparsity pattern of the reduced matrices applying the DCBM using 5 normal modes per substructure

In contrast the sparsity pattern of the reduced stiffness matrix $\boldsymbol{K}_{\text {red }}$ and the reduced mass matrix 
$\boldsymbol{M}_{\text {red }}$ obtained by the MNM and the RM, respectively, show full matrices. The MNM gives indeed an entirely diagonal reduced mass matrix $\boldsymbol{M}_{\text {red,MN }}$ (Fig. 5b) but causes always a full coupling between all DOF of all substructures via the reduced stiffness matrix $\boldsymbol{K}_{\text {red,MN }}$ (Fig. 5a). This makes the reusability of reduced models obtained by the MNM very inefficient and therefore nearly impossible from a practical point of view. The RM also causes a coupling between the substructures via interface displacements $\boldsymbol{u}_{b}$ in the reduced stiffness matrix $\boldsymbol{K}_{r e d, R}$ (Fig. 6a) as well as in the reduced mass $\boldsymbol{M}_{\text {red,R }}$ (Fig. 6b).

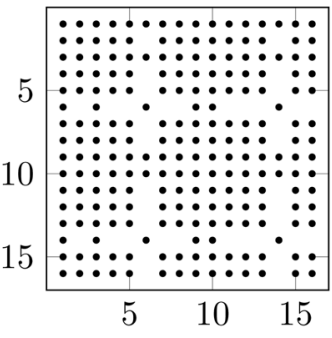

a) $\boldsymbol{K}_{r e d, M N}$

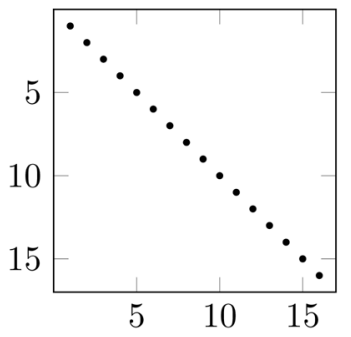

b) $M_{r e d, M N}$
Fig. 5. Sparsity pattern of the reduced matrices applying the MNM using 5 normal modes per substructure

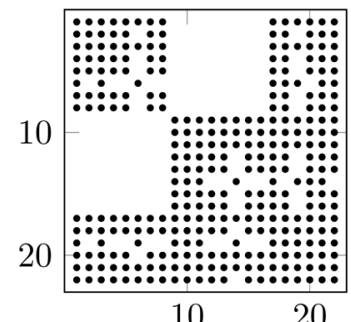

a) $\boldsymbol{K}_{\text {red,R }}$

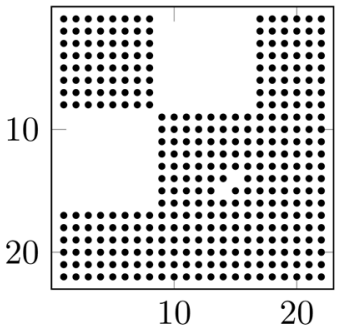

b) $M_{r e d, R}$
Fig. 6. Sparsity pattern of the reduced matrices applying the $R M$ using 5 normal modes per substructure

Moreover all DOF belonging to one reduced substructure are coupled with all other DOF of the same substructure which is why the reduced matrices of the RM are full for the substructure blocks and not diagonal. This result concerning the sparsity of the reduced matrices is outlined in Table 1 which shows the number $n$ of non-zero elements in the reduced matrices $\boldsymbol{K}_{r e d}$ and $\boldsymbol{M}_{\text {red }}$ and the sum $n_{\text {total }}$ of both obtained by the different methods for this example.

The reduced matrices of the CBM, the MNM and the DCBM contain a similar number of entries while the RM causes even for such a simple example a remarkable high number of entries. The number of entries of the MNM are comparable to the CBM and the DCBM but will increase dramatically if the number of substructures is increased since $\boldsymbol{K}_{\text {red }}$ will always be completely full.

Table 1. Number $n$ of non-zero elements in the reduced matrices obtained by the different methods for the Benfield truss ( 5 normal modes per substructure)

\begin{tabular}{lrrrc}
\hline & CBM & MNM & RM & DCBM \\
\hline$n$ in $\boldsymbol{K}_{\text {red }}$ & 40 & 216 & 314 & 196 \\
\hline$n$ in $\boldsymbol{M}_{\text {red }}$ & 118 & 16 & 354 & 50 \\
\hline$n_{\text {total }}$ & 158 & 232 & 668 & 246 \\
\hline
\end{tabular}

\subsection{Beam Frame}

In [6] a three-dimensional frame made of steel beams (Young's modulus $210 \mathrm{GPa}$, Poisson's ratio 0.3, and density $7500 \mathrm{kgm}^{3}$ ) schematically shown in Fig. 7 is considered. Each cell in the frame has a height of $0.35 \mathrm{~m}$ and a width and depth of $0.5 \mathrm{~m}$. All outer beams have a hollow circular cross-section with the outside and inside diameters $0.02 \mathrm{~m}$ and $0.018 \mathrm{~m}$. The diagonal members inside the cells have a solid circular cross section with diameter $0.008 \mathrm{~m}$. The frame is divided into 5 substructures and again the objective is the approximation of the lowest eigenfrequencies $\omega$ of the frame. Approximation of the eigenfrequencies of this system using the four presented methods with 4 normal modes per substructure (rigid body modes are not counted as free interface normal modes) is carried out. The results presented in [6] for the DCBM were obtained based erroneously on an incomplete set of free interface modes in the substructures using a simple Lanczos eigensolver: modes related to multiple frequencies were not determined by the simple Lanczos algorithm applied in that work.

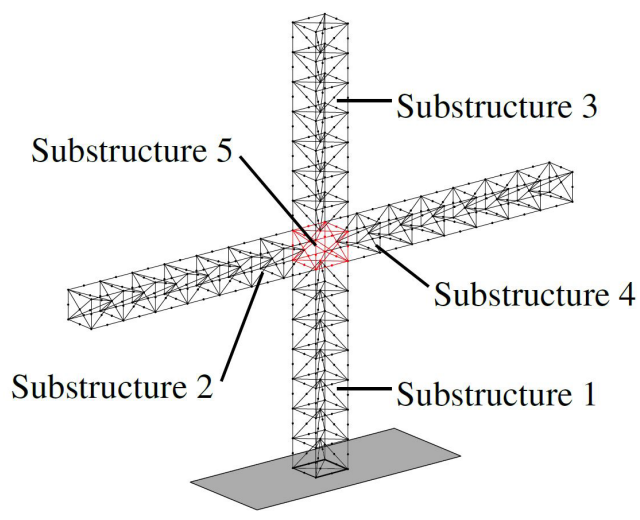

Fig. 7. Frame made of steel beams divided into 5 substructures [6] 
Considering the geometry of the beam frame structure of the arms in Fig. 7, the symmetry of the substructures is identifiable resulting in multiple eigenfrequencies of the substructures. The simple Lanczos eigensolver (no blocks, no restarts) used in [6] was not capable of determining multiple eigenvalues reliably [11]. Hence, when the simple Lanczos algorithm is used to determine the free modes of the substructure, modes that are multiple are missing and the representation of the lower spectrum is incomplete. Normal modes associated to the higher eigenfrequencies computed by the simple Lanczos eigensolver are used leading to poor approximation accuracy. Using the block Lanczos method and taking the lowest eigenfrequencies with associated subspaces of eigenvectors, the accuracy of the eigenfrequencies of the reduced global system obtained by the two reduction methods increases in the low frequency range [11]. In this work we are using an eigensolver capable of determining multiple eigenvalues for the comparison of the results obtained by the different methods. Again the eigenfrequencies $\omega_{\text {red }}$ of the reduced system applying the CBM, the MNM and the DCBM as well as the eigenfrequencies $\omega_{\text {full }}$ of the full (non-reduced) system are computed. The eigenfrequency relative errors $\varepsilon_{\text {rel }, j}=\left|\omega_{\text {red }, j}-\omega_{\text {full }, j}\right| / \omega_{\text {full }, j} \quad$ comparing the eigenfrequencies of the non-reduced model with the eigenfrequencies $\omega_{\text {red }}$ of the reduced system obtained by the reduction methods are plotted in Fig. 8. Nevertheless, as already observed in [6], this confirms that the accuracy of the DCBM in the low frequency

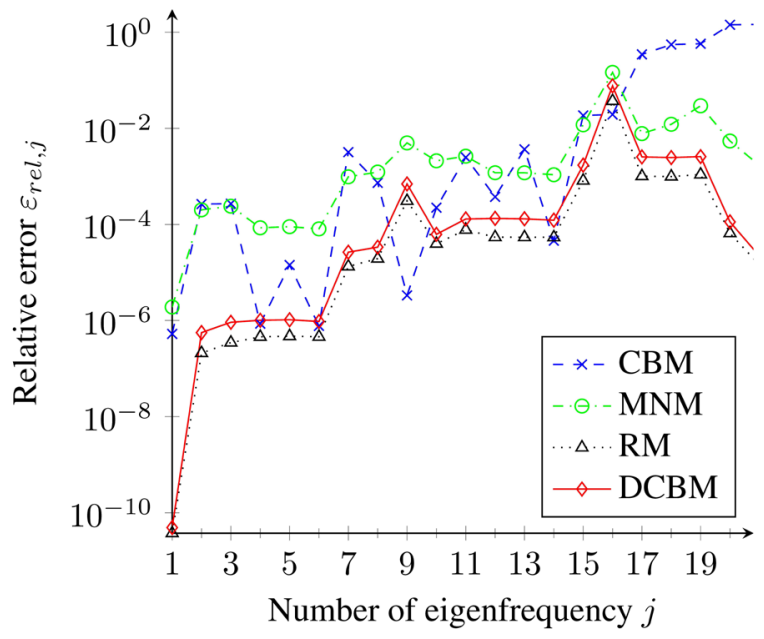

Fig. 8. Relative error $\varepsilon_{r e l, j}$ of eigenfrequency $j$ using 4 normal modes per substructure for the approximation of the lowest eigenfrequencies of the entire beam frame range is two orders of magnitude better than the CBM. Comparing only the free interface methods, the RM performs slightly better than the DCBM. Both the RM and DCBM give a much better approximation accuracy than the MNM.

\subsection{Two-dimensional solid}

In order to emphasize the weak coupling between the substructure interfaces applying the DCBM, as described in section 2.2.3, the problem of a two dimensional rectangle (density $\rho$, Young's modulus $E$, Poisson's ratio $v=0.3$ ) decomposed in 12 substructures as illustrated in Fig. 9 is considered. Each substructure is discretized by $16 \times 9$ bilinear four-noded elements (plane stress) and the structure is clamped on the left side in both directions. Again the objective is to approximate the lowest eigenfrequencies $\omega$ of the entire structure with the four different methods. Using 8 normal modes per substructure (not including potential rigid body modes) the relative errors $\varepsilon_{\text {rel }, j}=\left|\omega_{\text {red }, j}-\omega_{\text {full }, j}\right| / \omega_{\text {full }, j}$ depicted in Fig. 10 are resulting.

Making use of such a large reduction basis the DCBM and the RM give excellent results in the low frequency range in comparison to the CBM and the MNM. Approximating the 20 lowest eigenfrequencies of the full structure, neither compatibility problems nor trouble with negative eigenfrequencies occur during the reduction process with the DCBM. Similar graphs depicting the relative errors $\varepsilon_{r e l}$ as in Fig. 10 are obtained using different numbers of normal modes per substructure for the four methods.

When considering a very small reduction basis for the substructures (using only a small number of normal modes), non-physical negative eigenvalues of the reduced problem show up in the low frequency

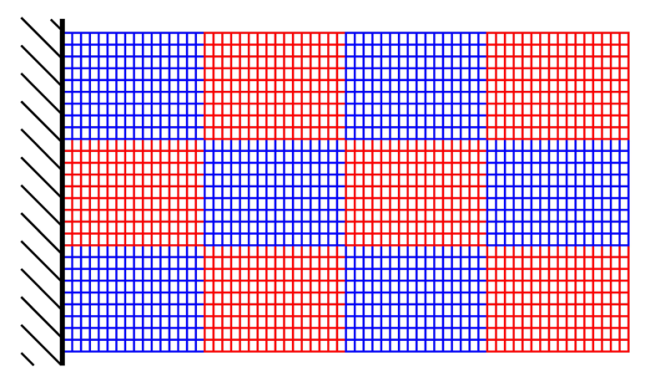

Fig. 9. Two dimensional solid problem (dimensionless width $l_{x}=16$, height $l_{y}=9$ decomposed in 12 substructures; each substructure is discretized by $16 \times 9$ bilinear four-noded elements (plane stress) and the structure is clamped on the left side in both directions 
range. Using only 2 normal modes per substructure as reduction basis for this example negative values emerge among the 20 smallest absolute values of eigensolutions $\omega^{2}$ applying the DCBM. In this case the first 14 of the lowest absolute values of $\omega^{2}$ are positive and the associated eigenmodes are approximating the true eigenvalues and eigenmodes of the full system accurately. But, as shown in Table 2, the $15^{\text {th }}$ eigenvalue is negative and the associated eigenmode depicted in Fig. 11 shows the non-physical behavior of this eigensolution.

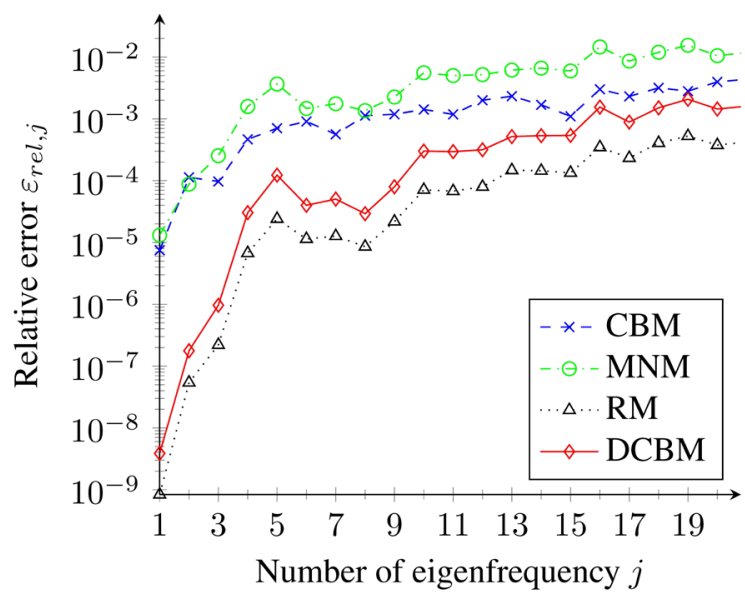

Fig. 10. Relative error $\varepsilon_{r e l, j}$ of eigenfrequency $j$ using 8 normal modes per substructure for the approximation of the lowest eigenfrequencies of the entire structure

Table 2. Number $j$ and associated eigenvalue $\omega_{j}^{2}$ of the two dimensional solid reduced with the DCBM

\begin{tabular}{cc}
\hline$j$ & $\omega_{j}^{2} \frac{\rho}{E}$ \\
\hline 1 & 0.000874 \\
\hline 2 & 0.009729 \\
\hline$\vdots$ & $\vdots$ \\
\hline 14 & 0.245011 \\
\hline 15 & -0.250785 \\
\hline 16 & 0.261676 \\
\hline
\end{tabular}

The eigenmodes corresponding to negative eigensolutions with higher absolute values show similar behavior. All these negative eigenvalues are related to the weak compatibility on the interfaces and not meaningful from a physical point of view. Consequently detecting and filtering out those negative eigenvalues is an additional step in the DCBM compared to the other methods based on primal assembly. This extra step is cheap and therefore does not increase the effort of the reduction process using the DCBM compared to the other four methods keeping the DCBM very efficient.

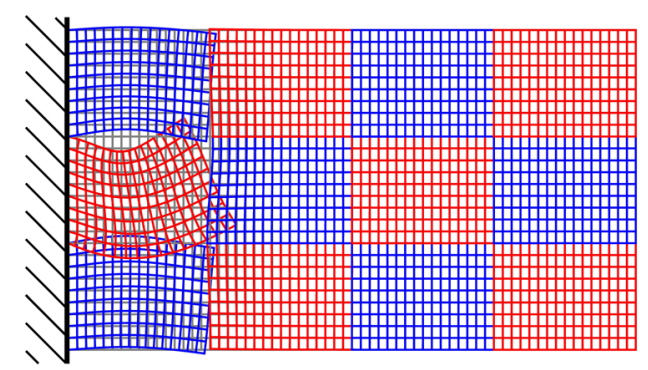

Fig. 11. Eigenmode number 15 approximated by the DCBM using 2 free interface normal modes per substructure

$$
\begin{gathered}
\left(\omega_{15}^{2}=-0.250785 \frac{E}{\rho}\right) \\
4 \text { CONCLUSIONS }
\end{gathered}
$$

\section{CONCLUSIONS}

In this paper the general concepts of the CraigBampton method (CBM), the MacNeal method (MNM), the Rubin method (RM) and the dual CraigBampton method (DCBM) were briefly presented, compared and discussed using three examples. The DCBM is outperforming the CBM using the same number of normal modes per substructure as reduction basis with comparable computational effort and having similar sparsity pattern of the reduced matrices. Comparing the free interface methods, the RM performs slightly better than the DCBM but results in full matrices. Both the RM and DCBM give a much better approximation accuracy than the MNM while the MNM generated always full coupled reduced matrices.

Properties of the DCBM were outlined and an additional necessary step, namely filtering out the negative eigensolutions, during the reduction process was illustrated. Non-physical negative eigenvalues of the reduced dual assembled problem are intrinsic in the reduction process using the DCBM caused by the weak compatibility on the interfaces between the substructures. Filtering out these negative eigenvalues is the decisive factor for the excellent approximation quality of the DCBM. The numerical effort adding this additional step is negligible keeping the efficiency of this method.

\section{REFERENCES}

[1] Craig, R.R. (2000). Coupling of substructures for dynamic analyses: An overview. Proceedings of AIAA/ASME/ASCE/ AHS/ASC Structures, Structural Dynamics, and Materials Conference and Exhibit, Atlanta, p. 1573-1584. 
[2] Craig, R.R., Bampton, M.C. (1968). Coupling of substructures for dynamic analyses. AIAA Journal, vol. 6, no. 7, p. 13131319, DOl:10.2514/3.4741.

[3] MacNeal, R.H. (1971). A hybrid method of component mode synthesis. Computers \& Structures, vol. 1, no. 4, p. 581-601, DOl:10.1016/0045-7949(71)90031-9.

[4] Rubin, S. (1975). Improved component-mode representation for structural dynamic analysis. AIAA Journal, vol. 13, no. 8, p. 995-1006, D0I:10.2514/3.60497.

[5] Herting, D.N. (1985). A general purpose, multi-stage, component modal synthesis method. Finite Elements in Analysis and Design, vol. 1, no. 2, p. 153-164, DOI:10.1016/0168-874X(85)90025-3.

[6] Rixen, D.J. (2004). A dual Craig-Bampton method for dynamic substructuring. Journal of Computational and Applied Mathematics, vol. 168, no. 1-2, p. 383-391, D0l:10.1016/j. cam.2003.12.014.

[7] Voormeeren, S.N., Van Der Valk, P.L.C., Rixen, D.J. (2011). Generalized methodology for assembly and reduction of component models for dynamic substructuring. AIAA Journal, vol. 49, no. 5, p. 1010-1020, D0l:10.2514/1.J050724.

[8] Craig, R.R., Chang, C.J. (1977). On the use of attachment modes in substructure coupling for dynamic analysis. $18^{\text {th }}$ Structural Dynamics and Materials Conference, San Diego, p. 89-99, Dol:10.2514/6.1977-405.

[9] Rixen, D.J. (2011). Interface Reduction in the Dual CraigBampton method based on dual interface modes. Linking Models and Experiments, vol. 2, Conference Proceedings of the Society for Experimental Mechanics Series, p. 311-328, DOI:10.1007/978-1-4419-9305-2_22.

[10] Benfield, W.A., Hruda, R.F. (1971). Vibration analysis of structures by component mode substitution. AIAA Journal, vol. 9, no. 7, p. 1255-1261, D0I:10.2514/3.49936.

[11] Gruber, F.M., Rutzmoser, J.B., Rixen, D.J. (2015). Comparison between primal and dual Craig-Bampton substructure reduction techniques. Proceedings of the 11th International Conference on Engineering Vibration, Ljubljana, p. 12451254. 